\title{
Imaging tumor angiogenesis
}

\author{
Kristy Red-Horse and Napoleone Ferrara
}

Genentech Inc., South San Francisco, California, USA.

\begin{abstract}
Since the discovery of vascular-specific growth factors with angiogenic activity, there has been a significant effort to develop cancer drugs that restrict tumorigenesis by targeting the blood supply. In this issue of the JCI, Mancuso et al. use mouse models to better understand the plasticity of the tumor vasculature in the face of antiangiogenic therapy (see the related article beginning on page 2610). They describe a rapid regrowth of the tumor vasculature following withdrawal of VEGFR inhibitors, emphasizing the importance of fully understanding the function of these and similar treatments used in the clinic at the cellular and molecular level.
\end{abstract}

Inhibiting tumor growth by limiting angiogenesis can, in combination with chemotherapy, extend life expectancy in patients with certain malignancies and is being heavily pursued as a promising cancer therapy (1). There are several FDAapproved antiangiogenenic drugs currently used in the clinic to treat cancer and neovascular age-related macular degeneration (bevacizumab, pegaptanib, ranibizumab, sunitinib, and sorafenib) and many others under development. These agents inhibit angiogenesis by targeting the signaling pathways mediated by VEGF-A and potentially other VEGF family members, such as placental growth factor (PlGF), VEGF-B, VEGF-C, and VEGF-D. However, VEGF-A is probably the major positive regulator of blood vessel formation. Given the success of anti-VEGF therapy in multiple mouse models of cancer and in clinical trials, it is important to fully understand the mechanisms by which anti-VEGF treatment affects tumor angiogenesis at the cellular and molecular level. This will be particularly important when designing combinatorial treatments aimed at circumventing drug resistance and tailoring patientspecific regimens.

In this issue of the JCI, Mancuso et al. further our understanding of how antiVEGF therapy affects blood vessels by describing the dynamics of tumor vasculature regrowth after drug withdrawal (2). Work from multiple laboratories (3) has established that inhibiting the VEGF pathway rapidly reduces the vascularity of solid tumors, leading to a decline in tumor

Conflict of interest: The authors are employed by Genentech Inc., manufacturer of bevacizumab.

Citation for this article: J. Clin. Invest. 116:2585-2587 (2006). doi:10.1172/JCI30058. growth. However, not all vessels are eliminated. A subset of VEGF-independent vessels expressing lower levels of VEGFR2 (4) remain, resulting in a more "normalized" vascular bed, no longer exhibiting the tortuosity and dysfunction frequently associated with tumor blood vessels. These remaining vessels could provide a source from which new vessels may bud following renewed VEGF activity. In addition, vascular regression leaves behind basement membrane sleeves - once associated with the endothelial cell layer - that can persist for up to 21 days (5). In previous work, McDonald and colleagues established that, in normal vascular beds such as the trachea and thyroid, regrowth following drug withdrawal is rapid and appears to be aided by the presence of basement membrane sleeves, identifying these structures as potential antiangiogenic targets $(4,6)$.

In their current report (2), the authors use elegant microscopic imaging techniques to extend these findings to angiogenesis associated with 2 tumor models, a transgenic mouse model of insulinoma (RIP-Tag2 tumors) and mice implanted with Lewis lung carcinoma xenografts. Two different small molecule tyrosine kinase inhibitors were used to target VEGFR activity: AG-013736 (7), which also blocks other receptor tyrosine kinases including PDGFR- $\beta$ and $c$-kit, and AG-028262, which has been reported to be a more selective inhibitor of VEGFR2 (8). Similar results were obtained using both drugs, suggesting that they function primarily through VEGF inhibition. The authors demonstrate that cessation of a 7-day therapy regimen is followed by rapid vascular regrowth, whereby the vessel density returned to pretreatment levels with- in 1 week (Figure 1). In accordance with previous findings $(4,6)$, posttreatment angiogenic growth correlated with the disappearance of empty basement membrane sleeves, suggesting that regrowth occurred along these structures (Figure 1). Attempts to prevent regrowth by blocking MMP activity with the MMP inhibitor AG3340 or blocking a cryptic site within type IV collagen with the monoclonal antibody HUIV26 were unsuccessful. However, this does not rule out the potential of regulating tumor angiogenesis by targeting these vascular relics.

\section{Role of basement membranes in the context of normal angiogenesis}

In the adult, the majority of angiogenesis is associated with wound healing, the reproductive tract, and inflammation. At the cellular level, this process occurs by several different mechanisms, including a phenomenon termed sprouting (9), which is a major method by which tumors recruit the preexisting vasculature. Sprouting is stimulated in response to local molecular cues, such as hypoxia- or inflammationinduced VEGF-A production. Thought to be a stepwise process, sprouting begins with local increases in vascular permeability, followed by basement membrane and ECM degradation. Subsequently, endothelial cells sometimes called tip cells send out projections and initiate migration along newly deposited ECM tracts. Finally, lumen-containing vessels are formed and integrated into the circulation. Recent evidence using real-time imaging techniques in zebrafish demonstrates that the latter steps involve, at least in some vessels, endothelial pinocytosis followed by coalescence of intracellular vacuoles (10).

At the molecular level, sprouting angiogenesis requires multiple factors, including MMPs such as MMP-2 (11) and MMP-9 (12), which function in ECM remodeling and in the liberation and modification of angiogenic factors such as VEGF-A and FGF (13). Integrins are also involved, as they bind sites within the ECM and are critical for endothelial cell proliferation, migration, and survival (14). In some cases, these 2 factors function in 


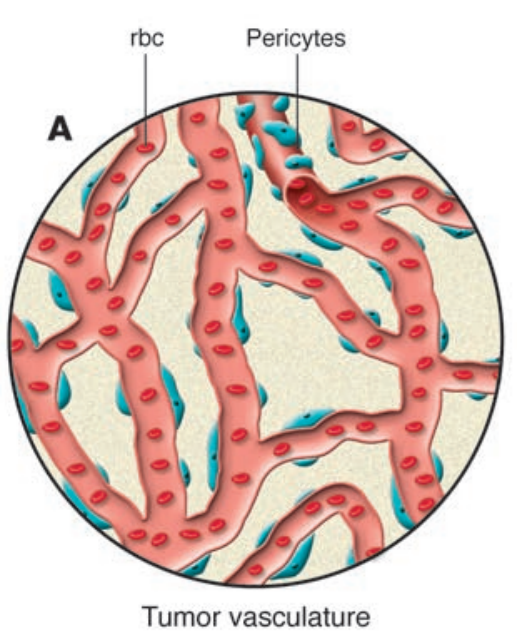

Tumor vasculature

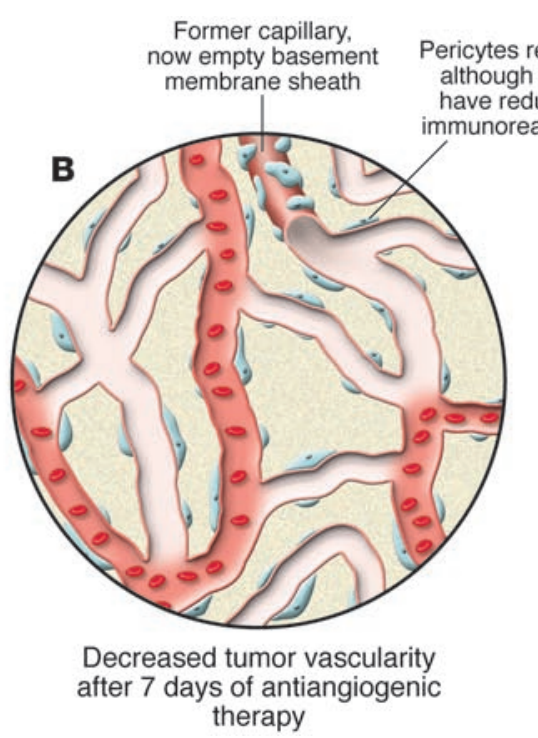

\section{Figure 1}

The effects of VEGF inhibition on the tumor vasculature are reversible. (A) Tumor growth stimulates angiogenesis, producing an abnormal vascular bed with disorganized branching and increased permeability. In many cases, this is due to increased VEGF production. (B) Antiangiogenic therapy that inhibits VEGF activity decreases tumor vascularity. Vascular regression often leaves behind a pericyte layer and empty basement membrane sleeves that can persist for up to 21 days. The pericytes were, however, observed to have reduced immunoreactivity to $\alpha-s m o o t h$ muscle actin. (C) Cessation of anti-VEGF therapy following a 7-day treatment regimen results in rapid vascular regrowth. Vessel density returns to pretreatment levels within 7 days.

the same pathway where MMPs cleave the ECM to expose cryptic angiogenenic integrin-binding sites $(15,16)$. Thus, blocking certain MMP and integrin family members can inhibit angiogenesis $(12,16)$. Mancuso et al. (2) similarly target MMPs and an integrin-binding site on type IV collagen in their system, but there is no effect on tumor revascularization. The difference between their model and others may be the presence of preassembled basement membrane sleeves that facilitate sprouting. In support of this theory, the authors localized VEGF to the basement membrane and show microscopic images that capture new vessel sprouts enveloped within the type IV collagen matrix. In addition, regrowth appears to occur along preexisting ECM tracts. Experiments analyzing regrowth in the presence and absence of these structures could address the issue; for example, examining the rate of angiogenesis in comparable vascular beds before and after the basement membrane has been degraded, which eventually occurs after longer treatment periods (5).

\section{Clinical implications of tumor revascularization}

What is the potential significance of rapid vascular regrowth to patients undergoing antiangiogenic therapy? Typically, in experimental models and clinical settings, treatment is given regularly for a sustained period of time, somewhat longer than that used in the current study (2). In addition, antiangiogenic therapy is combined with treatment with other cytotoxic agents. Thus, to answer this question, future work should focus on assessing the dynamics of the tumor vasculature following prolonged VEGF inhibition as well as after combination therapy. The imaging analysis described in this manuscript could also be extended to human tumor biopsy samples, perhaps leading to a method for monitoring patient responses to anti-VEGF therapy.

Despite it successes, antiangiogenic therapy is continually evolving, with the goal of developing the most effective strategies for different types of cancers and for each individual case. Current focuses are on establishing optimal dosages and combinations as well as identifying mechanisms of drug resistance. As to the former, data from experimental models and clinical trials indicate that the optimal anti-VEGF drug dosage may be critical to getting the maximum synergistic effects between it and chemotherapy or radiation (17). It has been suggested that inhibiting VEGF signaling "normalizes" the tumor vasculature, resulting in improved delivery and efficacy of chemotherapeutics and radiation (18-20). The quantitative imaging techniques well established by the authors should be helpful in addressing these and other issues by defining the tumor vasculature during critical stages of anti-VEGF treatment. Similarly, it may be informative to assess vasculature growth at the precise time when tumors begin to acquire resistance to antiangiogenic drugs. Resistance is associated with reestablishment of the tumor vasculature due to compensatory upregulation of other angiogenic factors such as FGF (21).

In summary, the work presented by Mancuso et al. (2) expands our knowledge of how targeting the VEGF pathway influences tumor angiogenesis. The rapid regrowth of tumor-associated vessels following derepression of VEGF signaling underscores the powerful angiogenic activity of this growth factor family. These and future studies aimed at broadening our understanding of antiangiogenenic therapy are important steps in furthering the development of this class of cancer therapeutics.

Address correspondence to: Napoleone Ferrara, Genentech Inc., 1 DNA Way, South San Francisco, California 94080, USA. Phone: (650) 225-2968; Fax: (650) 2256327; E-mail: nf@gene.com. 
1. Ferrara, N., and Kerbel, R.S. 2005. Angiogenesis as a therapeutic target. Nature. 438:967-974.

2. Mancuso, M.R., et al. 2006. Rapid vascular regrowth in tumors after reversal of VEGF inhibition. J. Clin. Invest. 116:2610-2621. doi:10.1172/JCI24612.

3. Gerber, H.P., and Ferrara, N. 2005. Pharmacology and pharmacodynamics of bevacizumab as monotherapy or in combination with cytotoxic therapy in preclinical studies. Cancer Res. 65:671-680.

4. Kamba, T., et al. 2006. VEGF-dependent plasticity of fenestrated capillaries in the normal adult microvasculature. Am. J. Physiol. Heart Circ. Physiol. 290:H560-H576.

5. Inai, T., et al. 2004. Inhibition of vascular endothelial growth factor (VEGF) signaling in cancer causes loss of endothelial fenestrations, regression of tumor vessels, and appearance of basement membrane ghosts. Am. J. Pathol. 165:35-52.

6. Baluk, P., et al. 2004. Regulated angiogenesis and vascular regression in mice overexpressing vascular endothelial growth factor in airways. Am. J. Pathol. 165:1071-1085.

7. Hu-Lowe, D., et al. 2002. Characterization of potency and activity of the VEGF/PDGF receptor tyrosine kinase inhibitor AG013736. Proc. Am.
Assoc. Cancer Res. 43:A5357.

8. Zou, H.Y., et al. 2004. AG-028262, a novel selective VEGFR tyrosine kinase antagonist that potently inhibits KDR signaling and angiogenesis in vitro and in vivo. Proc. Am. Assoc. Cancer Res. 45:A2578.

9. Conway, E.M., Collen, D., and Carmeliet, P. 2001. Molecular mechanisms of blood vessel growth. Cardiovasc Res. 49:507-521.

10. Kamei, M., et al. 2006. Endothelial tubes assemble from intracellular vacuoles in vivo. Nature. 442:453-456

11. Itoh, T., et al. 1998. Reduced angiogenesis and tumor progression in gelatinase A-deficient mice. Cancer Res. 58:1048-1051.

12. Bergers, G., et al. 2000. Matrix metalloproteinase-9 triggers the angiogenic switch during carcinogenesis. Nat. Cell Biol. 2:737-744.

13. Mott, J.D., and Werb, Z. 2004. Regulation of matrix biology by matrix metalloproteinases. Curr. Opin. Cell Biol. 16:558-564.

14. Stupack, D.G., and Cheresh, D.A. 2004. Integrins and angiogenesis. Curr. Top. Dev. Biol. 64:207-238.

15. Hangai, M., et al. 2002. Matrix metalloproteinase-9 dependent exposure of a cryptic migratory control site in collagen is required before retinal angiogen- esis. Am. J. Pathol. 161:1429-1437.

16. Xu, J., et al. 2001. Proteolytic exposure of a cryptic site within collagen type IV is required for angiogenesis and tumor growth in vivo. J. Cell Biol. 154:1069-1079.

17. Bergsland, E., and Dickler, M.N. 2004. Maximizing the potential of bevacizumab in cancer treatment. Oncologist. 9(Suppl. 1):36-42.

18. Winkler, F., et al. 2004. Kinetics of vascular normalization by VEGFR2 blockade governs brain tumor response to radiation: role of oxygenation, angiopoietin-1, and matrix metalloproteinases. Cancer Cell. 6:553-563.

19. Tong, R.T., et al. 2004. Vascular normalization by vascular endothelial growth factor receptor 2 blockade induces a pressure gradient across the vasculature and improves drug penetration in tumors. Cancer Res. 64:3731-3736.

20. Jain, R.K. 2005. Normalization of tumor vasculature: an emerging concept in antiangiogenic therapy. Science. 307:58-62.

21. Casanovas, O., Hicklin, D.J., Bergers, G., and Hanahan, D. 2005. Drug resistance by evasion of antiangiogenic targeting of VEGF signaling in late-stage pancreatic islet tumors. Cancer Cell. 8:299-309.

\title{
Myeloid suppressor cells regulate the adaptive immune response to cancer
}

\author{
Alan B. Frey \\ Department of Cell Biology, New York University School of Medicine, New York, New York, USA.
}

\begin{abstract}
Inflammation resultant from tumor growth, infection with certain pathogens, or in some cases, trauma, can result in systemic release of cytokines, especially GM-CSF, that in turn stimulate the abundant production and activation of a population of immature myeloid cells, termed myeloid suppressor cells (MSCs), that have potent immunosuppressive functions. In this issue of the JCI, Gallina and colleagues have illuminated some complex issues concerning the development, activation, and function of MSCs (see the related article beginning on page 2777). They show that activation of MSCs is initiated in response to IFN- $\gamma$, presumably produced in situ by antitumor $T$ cells in the tumor microenvironment. After this triggering event, MSCs express 2 enzymes involved in L-arginine metabolism, Arginase I and iNOS, whose metabolic products include diffusible and highly reactive peroxynitrites, the ultimate biochemical mediators of $T$ cell immune suppression. The multifaceted regulation of this complex suppressive effector system provides several potential therapeutic targets.
\end{abstract}

Immune response to tumor growth

As shown elegantly by North, priming of the adaptive immune response occurs during the early stage of tumor growth and results in development of $\mathrm{CD}^{+} \mathrm{T}$ cells reactive to tumors (1). Despite evident host

Nonstandard abbreviations used: IL-4R $\alpha$, IL-4 receptor $\alpha$; L-Arg, L-arginine; MSC, myeloid suppressor cell.

Conflict of interest: The author has declared that no conflict of interest exists.

Citation for this article: J. Clin. Invest. 116:2587-2590 (2006). doi:10.1172/JCI29906. recognition of tumor antigens, coincident with or immediately subsequent to $T$ cell priming, the antitumor immune response is inadequate to eliminate the tumor and is eventually dampened, thereby leading to tumor escape. Understanding how cancer growth affects the antitumor immune response and discovering how escape from antitumor immunity can be reversed are major goals in tumor immunology.

Several non-mutually exclusive phenomena are likely to contribute to inhibition of effective antitumor $\mathrm{T}$ cell immunity, including activation of Tregs (2), cancer "immunoediting" (3), direct tumor downregulation of the effector phase $(4,5)$, and activation of non-T cell suppressor cells (6). Myelopoiesis is often dramatically stimulated during tumor growth, resulting in accumulation (in secondary lymphoid organs, blood, and tumor tissue) and activation of a population of myeloid suppressor cells (MSCs). In mice, removal of the primary tumor results in normalization of the number of systemic MSCs, revealing a causal role for tumor growth in their aberrant accrual (7). Purified MSCs have been shown to inhibit both $\mathrm{CD}^{+}$and $\mathrm{CD}^{+} \mathrm{T}$ cell responses in vitro, and indirect but compelling studies imply that these cells can downregulate $\mathrm{T}$ cell functions in vivo.

\section{MSCs in cancer}

Recent research has focused on deciphering the role played by MSCs in the subversion, inhibition, or downregulation of the immune response to cancer (reviewed in ref. 6). MSCs are uniformly CD $11 \mathrm{~b}^{+} \mathrm{TCR}^{-}$ but express surface markers indicative of a mix of partially differentiated monocytes and DCs. The exact differentiation status of MSCs probably reflects the mechanism 